\title{
Residential Treatment as a Rehabilitation Opportunity for Alcoholism Care
}

\author{
Giorgio Cerizza and M. Paola Ranalletti*
}

Alcoholism Rehabilitation Unit of Rivolta d'Adda, S. Marta Hospital, Rivolta d'Adda - "Ospedale Maggiore" Crema, Italy

\begin{abstract}
Short residential rehabilitation in the treatment of alcohol dependence in Italy is becoming more widespread and significant.

This type of treatment offers the possibility, in a situation alcohol and drug free, to make a more accurate diagnosis and provide information relevant for the purposes of outpatient rehabilitation.

In addition, psychotherapy group allows the breaking of loneliness and the reactivation of interpersonal skills.

Taking care of the entire family helps the reactivation of the roles within the family.

The teams working in the residential rehabilitation program are multi-professional. This allows to take care of the patients from a psychological point of view, but also a medical and educational ones.
\end{abstract}

Keywords: Alcoholism, Alcohol-related disorders, Residential rehabilitation, Addiction, Multi-professional team.

\section{INTRODUCTION}

Talking about residential treatment for mental health problems has been, for many years, very difficult in Italy, to the extent of feeling guilty for it.

Lex 180 (the law which imposed the closure of asylums, settled on mandatory medical treatment and establishing the public mental health services), because of a certain ideological stance, it was understood as being incorrect maintaining residential places where one could take care, listen and understand mental diseases [1].

In spite of this point of view, we believe that the meaning of taking care is keeping time for listening, observing and understanding, all this requires residential structures.

Despite this adverse view, operators confident in their work and attentive to patients' needs, have experienced residential rehabilitation treatment centered on listening and understanding and were able to provide useful therapeutic support regarding the "pain of living." This has occurred in the treatment of psychiatric as well as addictions cases.

In psychiatry, the opportunity was reserved to an elite group of patients only. Same opportunity is not provided in public health care were law provides hospital wards of diagnosis and treatment (SPDC) which often look like hellish places.

Addictions, substance use and eating disorders are not only the evidence of bad habits or mere physical dependency but, in line with WHO's most recent statements, indicators

\footnotetext{
*Address correspondence to this author at the Alcoholism Rehabilitation Unit of Rivolta d'Adda, S. Marta Hospital, Rivolta d'Adda - "Ospedale Maggiore" Crema, Italy; Tel: 0039 0363378243; 0039 3475439934;

E-mail: noa.hrivolta@hcrema.it
}

of a strong existential uneasiness that has many similarities with the above mentioned "pain of living". In fact, common experience of those who deal with addicts, is to see how patients enter into the state of abstinence and start feeling a malaise which the use of substances was masking [2].

It is known that a fair amount of dependencies (between 30 and $50 \%$ ) points out a concomitant psychiatric disorder (disorders on axis I) and an even greater number of relevant behavioral disorders (diagnoses on Axis II).

Here below it is reported the experience of a twenty years old young man who came to the center with a diagnosis of epilepsy: G. comes along with his parents who are worried about the problems of their son. The guy is described as a quiet and introverted young man, attentive and respectful at school who, in a very short time, starts behaving erratically and enters into drinking habits.

Only after a few interviews the young man is admitted to our hospital and his trust in us start increasing. After about one week in hospital where the boy shows interaction problems and tendency to withdraw, G. starts showing, at first in individual setting and then also in group situations, paranoid tendencies and hallucinations which alcohol abuse had hidden for years. The boy had been previously evaluated by two different psychiatrists in two meetings of thirty minutes each, then total absence of any psychiatric disorder was decreed.

A suitable place and sufficient time are very important for a proper understanding of each single case.

To do this, we must be ready to adjust our planning and our space in order to allow the emergence of the meanings which we can only find when the subjective time is mature. In other words, it is impossible to think of a time which unifies, in a conventional manner, the time of a bipolar 
patient with his fluctuations, the time of a depressed person with its motionlessness or a cocaine addict with its excitement. All these symptoms may be the manifestation of similar existential diseases, which require a subjective time to be managed.

In fact the first element of a psychiatric diagnostic evaluation is based on assessing how subjective time is superimposed on the conventional time... The duration of an outpatient interview (45 '), but also the same mode of any clinic organization, with its deadlines and often its impersonal places, does not allow to understand the patient's real needs or to evaluate the effectiveness of the prescribed treatment. It becomes important to think over what should be the suitable time for that specific person or for that particular family unit. The work in a residential premise allows a continuous caring time, but one should apply his mind also on Spaces: it is common experience that the places are lived differently depending on the moods. A suitable place for a rehabilitation treatment must be able to expand, shrink, adapting itself to the situation the patient is experiencing. Would this possible in an outpatient clinic? Residential facilities for addicts should suit the multiple needs of the rehabilitation care: in order to manage alcohol-related diseases, restraints places (like hospital facilities) in the first period are required, while for group activities and management of common daily activities, spacious and comfortable places should be available. The environment fit to provide patients various opportunities and experiences (bars, gyms, cinemas, theaters, supermarkets etc..) and to keep in touch with the outside reality. The eclecticism of facilities make different activities possible (sports, psychomotricity, expressive psychotherapy, medical, recreational, etc.) and provides opportunities for the coming forth of informal moments.

\section{LISTENING AND OBSERVING}

It is impossible to start a rehabilitation program for alcoholism without understanding the particular relationship established by the patient with alcohol. It is often a significant and special relationship, which follows lived and fantasized moments. If we try to describe it as a human relation, we could easily compare it to a love story. The masked and silent dialogue of this bond cannot be captured in a rigorous setting [3]. Staff dealing with inmates should have knowledge of involved listening and observation (quite an extinct technique in local services) because this allows to collect a variety of information and a plurality of perspectives. We can say that every single moment spent in the host structure has great significance. This highly specialized clinical work, makes the taking charge of the patient in a continuum possible, and brings us closer to understand how a person "works", how he interacts with what is happening and more importantly, what was the substance role in his life. It may has been a bond of affection and support (as often occurs in people with low self-esteem, which have features or depressive personality disorder), it may has represented both the self-healing response to an underlying psychiatric disorder (hallucinations, delusions, paranoia) or to deal with moods difficult to manage otherwise (bereavement, relationship breakdown, violence).
To give an example, we report now the experience related with a 45 years old capable business manager: the lady accepted to be hospitalized, but her strong feeling of defeat for nearly three weeks seemed to invalidate the therapeutic process. Unexpectedly, in a psychotherapy group, a sexual abuse by a family member occurred when she was a little girl, was recalled by the patient. After this revelation her psychotherapeutic path start becoming very fruitful. What we believed to be an "unproductive" time for the therapy was, on the contrary, absolutely necessary to reach the critical time for the change to take place. Residency does not restrict listening and observing to the standard 45 ', time which can be optimal when the therapeutic relationship is already established, but totally inadequate when someone has to be helped to deal with such a difficult emotional detachment.

We can remove alcohol only if we can establish new important and trusting relationships, including the therapeutic one [4].

Only in informal settings we can achieve a true knowledge of the subject and lay the foundation for a relationship which is already looking beyond the diagnostic path towards the therapeutic alliance. When a relationship is Traduci da: ingleseDigita il testo o l'indirizzo di un sito web oppure traduci un documento.Annullatradotto automaticamente da Google Alphaauthentic, confident and true, masking is no longer necessary. It is commonly recommended not to insert paranoid patients into groups. However, we can say that the insertion of such patients in informal groups guaranteed by the presence of operators is possible and suggested since it allows to relate in a situation experienced as non-judgmental along with the sedimentation of moments of pleasure and closeness. The operators of a residential structure for alcoholism must be able to promote informal moments exactly with the same care of a doctor prescribing medicine. To achieve this competency proper training and the removal of cultural bias are very important. In fact, till now, it is common opinion that the closeness between therapists and the patients is a risk for the therapeutic outcome. This bias comes from a restrictive concept of the psychoanalytic setting and still influences most of the therapeutic interventions in many services. The characteristics of a residential rehabilitation setting, on the contrary, make the sharing of real experiences and emotions lived together possible. Therefore, in real time, there is the opportunity to think over with the patient on what is happening and, if necessary, give useful interpretations. Certainly, as we know, managing a therapeutic relationship in a so (un) structured setting is very different from an outpatient setting, at the same time important is the therapist's willingness to live the relationship in an informal way which means also accepting of being "used" by the patient according to his needs. The patient can experience with us different situations in respect of what he has previously undergone and therefore the possibility for us to become new models. This practice needs a special training and appropriate therapeutic use of informality, which is based on theoretical assumptions very different from traditional ones. 


\section{DIAGNOSIS AND TREATMENT PLANNING}

As diagnosis we mean that process which opens to the cause of the special relationship of the person with the substance and how this report fits into the patient relational world. According to encoded diagnostic criteria, we cannot really understand the particular state of being which induces the pathological bond with the substance, the illusion of "salvation" ("... alcohol helps me ......." ). Indeed, the risk is to disperse important information related with the family background and the social context which in a residential diagnostic process one can collect. The use of alcohol is an indicator of a wider discomfort. Taking charge of an alcoholic leads to take charge of the entire family. The diagnosis cannot be detached from observing and listening as the entire family system moves in therapeutic structured moments, but also and above all in informal moments.

It is evident that the diagnostic time, thus conceived, gives many opportunities for an individualized treatment planning which is planned in continuity with it.

Constant observation gives useful indications for an individualized treatment and avoid the general administration of drug protocols (substitute drugs) that are unfortunately the most common response of the Services. The residential is extremely useful at the beginning of a rehabilitation treatment because it allows a detailed study in an alcohol and drug free situation. The residency is also an excellent opportunity to break the seclusion and to restore relational connections by taking charge of the entire family [5]. The motivations given by living in group promote a fundamental "emotional-relational awakening ". The experiment in everyday life, even in tasks involving responsibilities, makes the restoration of family roles possible and interrupts delegating behavior. As residential therapists, we have understood that a therapeutic relationship "substitute drug", is fundamental and it should be offered as long as required. Alcohol-related disorders, psychiatric disorders, eating disorders, social estrangement need a multiplicity of possible responses and modulation of the intervention. The residential offers a possible answer to various forms of distress at different times of the caring of the patient. Starting from the engaging and the definition of the program to the management of the effects of redefinition therapeutic targets, but also as a moment of recovery where the motivation had failed.

\section{CONCLUSIONS}

The Service offers a residential treatment for alcoholrelated problems and others addictions. It is a public service. The admissions are planned previous to family interviews.

\section{THE MAIN CHARACTERISTICS}

\section{Motivational Function}

Construction of a motivation by experiencing meaningful relational occasions in "alcohol-free" situation.

\section{Evaluation Function}

Accurate assessment to allow a diagnosis of the patient and / or of the family set up.

\section{FEATURE PROJECT}

Construction of an individualized treatment plan which may be tested and monitored during the hospitalization to establish precise intervention and a successful engagement with local structures.

The therapeutic tools shared by the entire team are: the participant observation as a constant search for understanding and the therapeutic relationship experienced as a healthy interaction and a welcoming and stimulating change.

The rehabilitative intervention aims at the understanding of why alcohol has been an irreplaceable resource. Only the answer to this question can let one understand by what the substance can be replaced. The therapeutic model of the "experiential Pleasantness"[6], stems from this observation and promises to experiment with alternative resources, new delights, authentic relationships that the person can make an informed choice.

\section{THE ACTIVITIES THAT UNDERPIN CLINICAL PRACTICE ARE}

JOB FAMILY: Dating multi family groups, family or couple interviews, support for parents, psychotherapy for couples, recreational activities with family in informal context.

\section{Communicative Area}

Individual psychotherapy and group activities with various ways of expression like Theatre Workshop, themebased groups, problem-solving groups, moments of spontaneous and informal conversation. BODY AREA: individualized programs on self-care, dancing, motor activity (gymnastics, psychomotor, biofeedback, etc...) Sports (swimming, running, soccer).

\section{Area of Autonomy and Restoration of Roles}

Daily care management of the environments, Activities aimed at cooperation and taking responsibility, personalized plans for economic management, outputs of group and individual allowances designed to experiment with nonsecure environment, preparatory activities for job searching.

\section{Area Medical Health}

Physician consultation and diagnostic evaluations, management of the withdrawal, exams and procedures aimed at evaluating diagnostic, prescribing and administering therapies / medical.

The hospital provides a personal involvement in the care of the guests and the reorganization of the department to emphasize the need to experiment with autonomy, to care for themselves and their environment. The proposed activities are designed to allow people to reflect on various aspects: physical conditions, the ability to relate to others, personal and family problems. Group is a very important resource for guests and a therapeutic tool for operators. The team is composed of three physicians, one psychiatrist (the Manager), one psychologist, a head nurse, 4 educators, 11 nurses and two medical social workers. 
A theater operator is constantly collaborating with the team and so does a football coach with his assistants.

\section{CONFLICT OF INTERESTS}

The authors confirm that this article content has no conflicts of interest.

\section{ACKNOWLEDGEMENT}

Declared none.

\section{REFERENCES}

[1] Cerizza G, Cibin M, Hinnenthal I, Ranalletti MP, Dondi E, Vittadini G. in Mission n 27/2008 "La residenzialità Riabilitativa Alcologica come opportunità terapeutica”, 2008.
[2] Hinnenthal I, Laki Z, Ardissone G. Psicotraumatologia e neuroplasticità. Presupposti teorici per la gestione clinica nel trattamento residenziale di alcolisti con poliabuso. In: Buone pratiche procedure terapeutiche nella gestione del paziente alcolista. Milano: Franco Angeli, 2008

[3] Cerizza G. Quando il limite diventa risorsa. Milano: F. Angeli, 1998.

[4] Bowlby. Costruzione e rottura dei legami affettivi. Maloino Raffaello Cortina 1982.

[5] Cerizza G, Greco C. Alcol e famiglia, in Il profilo professionale dell'alcologo. Lucchini A, Greco C, Cerizza G. Milano: F. Angeli, 1999.

[6] AAVV in Mission $n^{\circ}$ 18/2006. Il modello della Piacevolezza Esperienziale, Ospedale Santa Marta di Rivolta d'Adda, Dalle premesse generali all'attuale strutturazione, 2006.

(C) Cerizza and Ranalletti; Licensee Bentham Open.

This is an open access article licensed under the terms of the Creative Commons Attribution Non-Commercial License (http://creativecommons.org/licenses/by$\mathrm{nc} / 3.0 /$ ), which permits unrestricted, non-commercial use, distribution and reproduction in any medium, provided the work is properly cited. 